\title{
LL-37 stimulates the functions of adipose- derived stromal/stem cells via early growth response 1 and the MAPK pathway
}

Yoolhee Yang ${ }^{1}$, Hyunju Choi ${ }^{1}$, Mira Seon ${ }^{1}$, Daeho Cho $^{2^{*+}}$ and Sa Ik Bang ${ }^{1,3^{*}+}$

\begin{abstract}
Background: LL-37 is a naturally occurring antimicrobial peptide found in the wound bed and assists wound repair. No published study has characterized the role of LL-37 in the function(s) of human mesenchymal stem cells (MSCs). This study investigated the functions of adipose-derived stromal/stem cells (ASCs) activated by LL-37 by performing both in vitro assays with cultured cells and in vivo assays with C57BL/6 mice with hair loss.

Methods: Human ASCs were isolated from healthy donors with written informed consent. To examine the effects of LL-37 on ASC function, cell proliferation and migration were measured by a cell counting kit (CCK-8) and a Transwell migration assay. Early growth response 1 (EGR1) mRNA expression was determined by microarray and real-time PCR analyses. The protein levels of EGR1 and regenerative factors were analyzed by specific enzyme-linked immunosorbent assays and western blotting.

Results: LL-37 treatment enhanced the proliferation and migration of human ASCs expressing formyl peptide receptor like-1. Microarray and real-time PCR data showed that EGR1 expression was rapidly and significantly increased by LL-37 treatment. LL-37 treatment also enhanced the production of EGR1. Moreover, small interfering RNA-mediated knockdown of EGR1 inhibited LL-37-enhanced ASC proliferation and migration. Activation of mitogen-activated protein kinases (MAPKs) was essential not only for LL-37-enhanced ASC proliferation and migration but also EGR1 expression; treatment with a specific inhibitor of extracellular signal-regulated kinase, p38, or c-Jun N-terminal kinase blocked the stimulatory effect of LL-37. EGR1 has a strong paracrine capability and can influence angiogenic factors in ASCs; therefore, we evaluated the secretion levels of vascular endothelial growth factor, thymosin beta-4, monocyte chemoattractant protein-1, and stromal cell-derived factor-1. LL-37 treatment increased the secretion of these regenerative factors. Moreover, treatment with the conditioned medium of ASCs pre-activated with LL-37 strongly promoted hair growth in vivo.
\end{abstract}

Conclusions: These findings show that LL-37 increases EGR1 expression and MAPK activation, and that preconditioning of ASCs with LL-37 has a strong potential to promote hair growth in vivo. This study correlates LL-37 with MSC functions (specifically those of ASCs), including cell expansion, cell migration, and paracrine actions, which may be useful in terms of implantation for tissue regeneration.

Keywords: LL-37, Mesenchymal stem cells, Adipose-derived stromal/stem cells, Early growth response 1, Cell migration, Proliferation, Paracrine actions, MAPK pathway, Hair growth, Regeneration

\footnotetext{
* Correspondence: cdhkor@sookmyung.ac.kr; si55.bang@samsung.com

${ }^{\dagger}$ Equal contributors

²Department of Life Science, Sookmyung Women's University, Seoul, Korea

${ }^{1}$ Department of Plastic Surgery, Samsung Medical Center, Sungkyunkwan

University School of Medicine, Seoul, Korea

Full list of author information is available at the end of the article
} 


\section{Background}

Cell therapy using adult multipotent stromal cells or mesenchymal stem cells (MSCs) is clinically used to repair and regenerate various damaged tissues [1, 2]. Adipose-derived stromal/stem cells (ASCs) are multipotent mesenchymal cells isolated from adipose tissue and are accessible, abundant, and self-replenishing [3]. These cells are also capable of differentiating into multiple mesenchymal lineages, including osteoblasts, adipocytes, chondrocytes, and other types of cells. The differentiation, proliferation, and direct migration of MSCs into local damaged tissue undergoing regeneration are regarded as the primary mechanisms underlying the actions of MSCs [3, 4]. Besides these actions, the strong paracrine effects of various growth factors and cytokines secreted by MSCs is a key mechanism underlying MSCmediated tissue regeneration and repair $[5,6]$. Therefore, it is important to understand the molecular mechanism controlling the machinery that underlies these strong paracrine effects in MSCs.

Early growth response 1 (EGR1), a member of the immediate-early gene family, encodes a zinc finger transcription factor and is rapidly induced by mitogens and growth factors [7]. Once induced, EGR1 plays a pivotal role in the expression and production of various growth factors, cytokines, and other bioactive molecules, resulting in the stimulation of cellular functions for tissue repair and regeneration. Interestingly, EGR1 might be an activation marker of human MSCs, which highly express the EGR1 gene, and have multiple roles involving angiogenesis and mitogenesis [8,9]. Strong induction of EGR1 is mediated by the mitogen-activated protein kinase (MAPK) pathway, a crucial signaling pathway associated with cell migration and proliferation $[7,10]$.

LL-37 is a naturally occurring 37-amino acid sequence synthesized from the C-terminus of human cationic antimicrobial protein 18 (hCAP-18) [11] and is widely found in various body fluids and cell types including epithelial cells and immune cells $[12,13]$. Secretion of LL$37 /$ hCAP-18 is significantly elevated at the wound bed, where this peptide demonstrates proliferative, angiogenic, and immunomodulatory activities through the MAPK pathway [14]. Besides participating in innate host defense $[11,15]$, this peptide also has wound-healing effects $[16,17]$ and is a potent chemoattractant for various cell types including immune cells through activation of formyl peptide receptor like-1 (FPRL1), its main receptor [18]. A recent study by Krasnodembskaya et al. showed that human MSCs possess direct antimicrobial activity, which is mediated in part by secretion of the human cathelicidin hCAP-18/LL-37 [19].

Many studies reported ASC-mediated tissue regeneration in various damaged tissues $[1,20]$ and LL-37 is an important mediator of the repair and regeneration of wounds, bones, islets, and other damaged tissues [16, 21, 22]. However, the precise effect of LL-37 on adjacent human ASCs has not been identified. In the present study, we hypothesized that LL-37 enhances their therapeutic potential by activating ASCs via EGR1 and MAPK signaling. Our findings indicate that LL-37 may be used as a preconditioning agent before ASC transplantation for tissue regeneration.

\section{Methods \\ Cell culture}

Subcutaneous adipose tissue was obtained during elective surgeries with written informed consent, as approved by the Samsung Medical Center Institutional Review Board. All donors were $<40$ years old and did not have diabetes or acute inflammation. The mean body mass index of the donors was $25.2 \pm 3.64$. Human ASCs were isolated according to a previous protocol [23] and cultured in lowglucose Dulbecco's modified Eagle's medium supplemented with $10 \%$ fetal bovine serum, $100 \mathrm{U} / \mathrm{mL}$ penicillin, and $100 \mu \mathrm{g} / \mathrm{mL}$ streptomycin at $37^{\circ} \mathrm{C}$ in a humidified atmosphere containing $5 \% \mathrm{CO}_{2}$. ASCs were characterized by the presence of the cell surface markers CD73, CD90, and CD105 and the absence of CD11b, CD34, CD45, and HLA-DR [24].

\section{Cell viability and proliferation assays}

Cells were treated with human LL-37 (Phoenix Pharmaceuticals, USA) for $48 \mathrm{hr}$ under serum deprivation conditions. Cell viability was determined by Trypan blue staining. Cell proliferation was measured with the cell counting kit (CCK)-8 according to the manufacturer's protocol (Dojindo, Japan). ASCs $\left(5 \times 10^{3}\right.$ cells/well) were treated with $2.5-20 \mu \mathrm{g} / \mathrm{mL}$ LL-37 for 24 and $48 \mathrm{hr}$ prior to adding CCK-8 solution. Absorbance at $450 \mathrm{~nm}$ was determined with a multi-plate reader (Molecular Devices, CA, USA).

\section{Migration assay}

A cell migration assay was performed using Transwell plates (8 $\mu \mathrm{m}$ pore size; Costar, Corning, NY, USA) according to a previous study [25]. Briefly, ASCs were suspended in serum-free medium and $100 \mu \mathrm{L}$ of the cell suspension $\left(7 \times 10^{5}\right.$ cells $\left./ \mathrm{mL}\right)$ was added to each upper well. LL-37 at the indicated concentrations $(5,10$, and $20 \mu \mathrm{g} / \mathrm{mL}$ ) was placed in the lower wells of a 24 -well tissue culture plate. After incubation for $6 \mathrm{hr}$ at $37^{\circ} \mathrm{C}$, cells that had migrated were stained with $0.15 \%$ crystal violet and counted in five random microscopy fields using the Scanscope scanning system (Aperio Scanscope, CA, USA).

\section{Small interfering RNA transfection}

Cells were transfected with EGR1-targeting small interfering RNA (siRNA) (Santa Cruz Biotechnology, USA) 
or negative control siRNA (Bioneer, Daejeon, Korea) using Lipofectamine RNAi (Invitrogen, CA, USA). Briefly, when cells reached 60-70\% confluency, siRNA (final concentration, $100 \mathrm{nM}$ ) was combined with Lipofectamine RNAi and allowed to complex for $20 \mathrm{~min}$. The transfection mixture was then applied to ASCs and incubated for $6 \mathrm{hr}$ at $37{ }^{\circ} \mathrm{C}$. Subsequently, cells were maintained in complete medium for $36 \mathrm{hr}$ before being subjected to the cell migration assay and enzyme-linked immunosorbent assays (ELISAs).

\section{Western blot analysis}

Total protein from cell lysates was separated by SDSPAGE and transferred to a nitrocellulose membrane, which was incubated with the corresponding primary antibodies (anti-EGR1 (Santa Cruz Biotechnology) and anti-GAPDH (Cell Signaling, USA)) overnight at $4{ }^{\circ} \mathrm{C}$. Washed membranes were incubated for $1 \mathrm{hr}$ with a horseradish peroxidase-conjugated anti-mouse secondary antibody. Bands were visualized using an enhanced chemiluminescence detection system (Amersham Biosciences, Piscataway, NJ, USA). The band intensities were quantified using TotalLab software (UK).

\section{Fluorescence-activated cell sorting}

FPRL1 expression in ASCs from four donors was evaluated by surface staining. Cells were washed with fluorescenceactivated cell sorting (FACS) buffer, stained with a mouse anti-FPRL1 antibody (R\&D Systems, USA), and then stained with anti-mouse IgG-FITC (Sigma, USA). Labeled cells were measured with a FACSCalibur instrument (Becton Dickinson Biosciences, CA, USA) and analyzed using the Win MDI program (Win MDI version 2.8).

\section{Immunostaining}

Immunofluorescence analysis was performed as previously described [23]. Briefly, ASCs $\left(5 \times 10^{3}\right.$ cells/well $)$ were seeded on four-well Lab-Tek II chamber slides (Nalge Nunc International, IL, USA), treated with LL-37 for $48 \mathrm{hr}$, fixed, and permeabilized for 20 min with $0.1 \%$ Triton X-100 prepared in phosphate-buffered saline. After washing, cells were blocked and incubated with an anti-proliferating cell nuclear antigen (PCNA) antibody (Abcam, Cambridgeshire, UK) for $1 \mathrm{hr}$. Next, cells were incubated with goat anti-mouse IgG-Alexa Fluor 488 (Invitrogen) for $30 \mathrm{~min}$ at $37{ }^{\circ} \mathrm{C}$. A nucleic acid dye (DAPI; $0.5 \mu \mathrm{g} / \mathrm{mL}$ ) was added to stain the nuclei. PCNA immunofluorescence was detected with an LSM700 confocal microscope system (Carl Zeiss, NY, USA; 400x objective).

\section{ELISA}

Cells were plated in six-well plates $(300,000$ cells/well) and treated with $20 \mu \mathrm{g} / \mathrm{mL} \mathrm{LL}-37$ in serum-free medium. After $48 \mathrm{hr}$, the culture medium was collected and ELISAs were performed for thymosin beta-4 (TB4; Immune Diagnostics, Canada), vascular endothelial growth factor (VEGF; Invitrogen), monocyte chemoattractant protein-1 (MCP-1; eBioscience, USA), stromal cell-derived factor-1 (SDF-1; RayBiotech, USA), and EGR1 (EIAab, China), in accordance with the manufacturers' recommendations. For detection of EGR1, the cells were lysed by the addition of $200 \mu \mathrm{L}$ of cell lysis buffer after LL-37 treatment for $6 \mathrm{hr}$.

\section{Real-time PCR}

Total RNA was extracted using TRIzol reagent (Invitrogen). After removing possible DNA contamination by DNAse treatment of the extracted RNA, cDNA was synthesized using $2 \mu \mathrm{g}$ of total RNA and SuperScript II reverse transcriptase according to the manufacturer's instructions. The primers used are provided in Additional file 1: Table S1. For real-time PCR, quantitative PCR was performed using the 7900 Real-Time PCR System (Applied Biosystems, Foster City, CA, USA) and the Power SYBR Green qPCR Master Mix Kit (Life Technologies, CA, USA). The cycling profile for real-time PCR $\left(50\right.$ cycles) was as follows: $95{ }^{\circ} \mathrm{C}$ for $10 \mathrm{~min}, 95{ }^{\circ} \mathrm{C}$ for $15 \mathrm{sec}$, and $60{ }^{\circ} \mathrm{C}$ for $60 \mathrm{sec}$. The comparative threshold cycle (Ct) method (i.e., $2-\Delta \Delta \mathrm{Ct}$ ) was used to calculate fold amplification.

\section{Microarray}

The microarray data are accessible in the Gene Expression Omnibus (GEO) database under accession number GSE76392. For the microarray, human ASCs were treated with $20 \mu \mathrm{g} / \mathrm{mL}$ LL-37 for $1 \mathrm{hr}$. Each total RNA sample (200 ng) was labeled and amplified using the Low Input Quick Amp Labeling Kit (Agilent Technologies, CA, USA). Cy3-labeled aRNAs were resuspended in $100 \mu \mathrm{L}$ of hybridization solution (Agilent Technologies). Labeled aRNAs were placed on the Agilent SurePrint G3 Human GE 4x44K array (Agilent Technologies) and covered with a Gasket 8-plex slide (Agilent Technologies). Slides were hybridized for $17 \mathrm{hr}$ at $65{ }^{\circ} \mathrm{C}$. The hybridized slides were sequentially washed in $2 \times$ SSC containing $0.1 \% \mathrm{SDS}$ for $2 \mathrm{~min}, 1 \times \mathrm{SSC}$ for $3 \mathrm{~min}$, and $0.2 \times \mathrm{SSC}$ for $2 \mathrm{~min}$ at room temperature. Finally, the slides were centrifuged at $3000 \mathrm{rpm}$ for $20 \mathrm{sec}$ to dry. Arrays were analyzed using an Agilent scanner with associated software. Gene expression levels were calculated with Feature Extraction v10.7.3.1 (Agilent Technologies). The relative signal intensities of each gene were generated using the Robust MultiArray Average algorithm. Data were processed based on the quantile normalization method using GeneSpring GX 13.0 (Agilent Technologies). This normalization method aims to make the distribution of intensities for each array in a set of arrays the same. The normalized and log- 
transformed intensity values were analyzed using GeneSpring GX 13.0. Fold-change filters included the requirement that the genes be present in at least $200 \%$ of controls for upregulated genes and less than $50 \%$ of controls for downregulated genes. Hierarchical cluster analysis was conducted using the Cluster 3.0 program, the Euclidean distance, and average linkage algorithm.

\section{Animals and in vivo hair growth test}

Five-week-old male C57BL/6 mice were purchased from SLC Inc. (Haruno, Japan) and allowed to adapt to their new environment for 2 weeks. The fur on the backs of 7 -week-old mice $(n=14)$ was shaved with hair clippers and removed with hair removal cream. Conditioned medium (CM) was topically applied daily for up to 18 days. Pigment darkening and the hair growth rate were monitored every 3 days for 2 weeks. Hair growth was evaluated by three independent dermatological scientists. The hair growth score was determined using the method described by Vegesna et al. [26, 27]. The animal experiments were approved by the Institutional Animal Care and Use Committee of Samsung Medical Center (approval number: SMC-IACUC-2013-0103-003) and all experiments followed regulatory standards.

\section{Plasmid construction and selection of stably transfected cell lines}

hEGR1 was subcloned into the Hind III/Xho I sites of pcDNA3.1(+). After digestion of the pcDNA3.1(+)hEGR1 plasmid with the restriction enzymes, the resulting $h E G R 1$ gene along with the BamHI and SfiI sites was inserted into the multiple cloning site of the expression plasmid pLenti6/V5-D-TOPO (Invitrogen), which contains a cytomegalovirus promoter upstream of the inserted gene. The resulting plasmid was named pLenti6/V5-hEGR1.

The lentiviral expression system based on four plasmids was obtained from Invitrogen. Briefly, $2.5 \mu \mathrm{g}$ of pLP1, $2.5 \mu \mathrm{g}$ of pLP2, $2.5 \mu \mathrm{g}$ of pLP/VSVG, and $2.5 \mu \mathrm{g}$ of the lentiviral vector pLenti6/V5-hCAMP were cotransfected with Fugene6 (Roche) into HEK293T cells cultured in $10 \mathrm{~cm}$ plates. The medium was changed every $24 \mathrm{hr}$. After $48 \mathrm{hr}$, supernatants were pooled, filtered through a $0.45-\mu \mathrm{m}$ filter, and centrifuged at $6000 \mathrm{rpm}$ at $4{ }^{\circ} \mathrm{C}$ for $16 \mathrm{hr}$. The pellet was resuspended in $1 \mathrm{~mL}$ of complete medium and stored at $-80^{\circ} \mathrm{C}$. The resulting recombinant lentivirus was added to ASCs cultured in $10 \mathrm{~cm}$ plates. At $48 \mathrm{hr}$ after seeding, the medium was replaced with complete medium containing $10 \mu \mathrm{g} / \mathrm{mL}$ blasticidin as a selective agent. At 18 days after transfection, selected cell colonies were split into $60 \mathrm{~mm}$ petri dishes. Isolated cell colonies were cultured and expanded.

\section{Statistical analysis}

Statistical significance was estimated using the Student's $t$-test. Mean differences were considered to be significant when $P<0.05$.

\section{Results}

LL-37 increases the proliferation and migration of human ASCs in a dose-dependent manner

LL-37 reportedly activates functions such as proliferation and migration through FPRL1, a G-protein-coupled receptor, in many cell types [18]; therefore, several donor pools of ASCs were examined for expression of FPRL1. Flow cytometric data confirmed that FPRL1 was expressed on ASCs in each donor pool (Fig. 1a).

To investigate the role of LL-37 in ASC proliferation, we examined the effect of LL-37 on the proliferative ability of ASCs using the CCK-8 assay. LL-37 treatment markedly stimulated ASC proliferation in a dosedependent manner without causing cytotoxicity (Fig. 1b and Additional file 2: Figure S1a). Furthermore, we determined the number of proliferating cells by performing immunofluorescence staining of PCNA, a nuclear protein associated with cell proliferation. The proportion of ASCs with positively stained nuclei (green fluorescence) was markedly enhanced by LL-37 treatment (Fig. 1c).

To investigate the effects of LL-37 on ASC migration, a Transwell migration assay was performed. LL-37 treatment rapidly increased human ASC migration within $6 \mathrm{hr}$; this effect was dose-dependent, with maximal stimulation at $20 \mu \mathrm{g} / \mathrm{mL}$ among the concentrations tested (Fig. 1d). ASCs were pretreated with pertussis toxin (Ptx), a Gai inhibitor, or a neutralizing anti-LL-37 antibody $(\alpha L L-37)$ before activation with LL-37. Ptx or $\alpha \mathrm{LL}-37$ treatment prior to LL-37 stimulation significantly inhibited ASC migration and proliferation (Fig. 1e and $\mathrm{f}$ ). Taken together, these data suggest that LL-37 induces ASC proliferation and migration through the Gai-coupled receptor FPRL1.

\section{EGR1 is critical for LL-37-enhanced ASC migration and proliferation}

We investigated the target genes of LL-37 that induce ASC proliferation and migration using the Agilent human $4 \times 44 K$ array, a human signaling pathway finder. Microarray analysis showed that LL-37 treatment significantly increased EGR1 expression by 18.47 -fold (Table 1 and Additional file 3: Table S2). LL-37 treatment also increased the levels of several genes (including EGR2, early growth response 2; KLF10, Krupple-like factor 10; FOS; and CTGF, connective tissue growth factor) linked to various functions, such as the cell cycle, cell migration, cell proliferation, and transcription. To confirm the effect of LL-37 treatment on EGR1 expression in ASCs, cells were treated with 10 or $20 \mu \mathrm{g} / \mathrm{mL}$ LL-37 for 


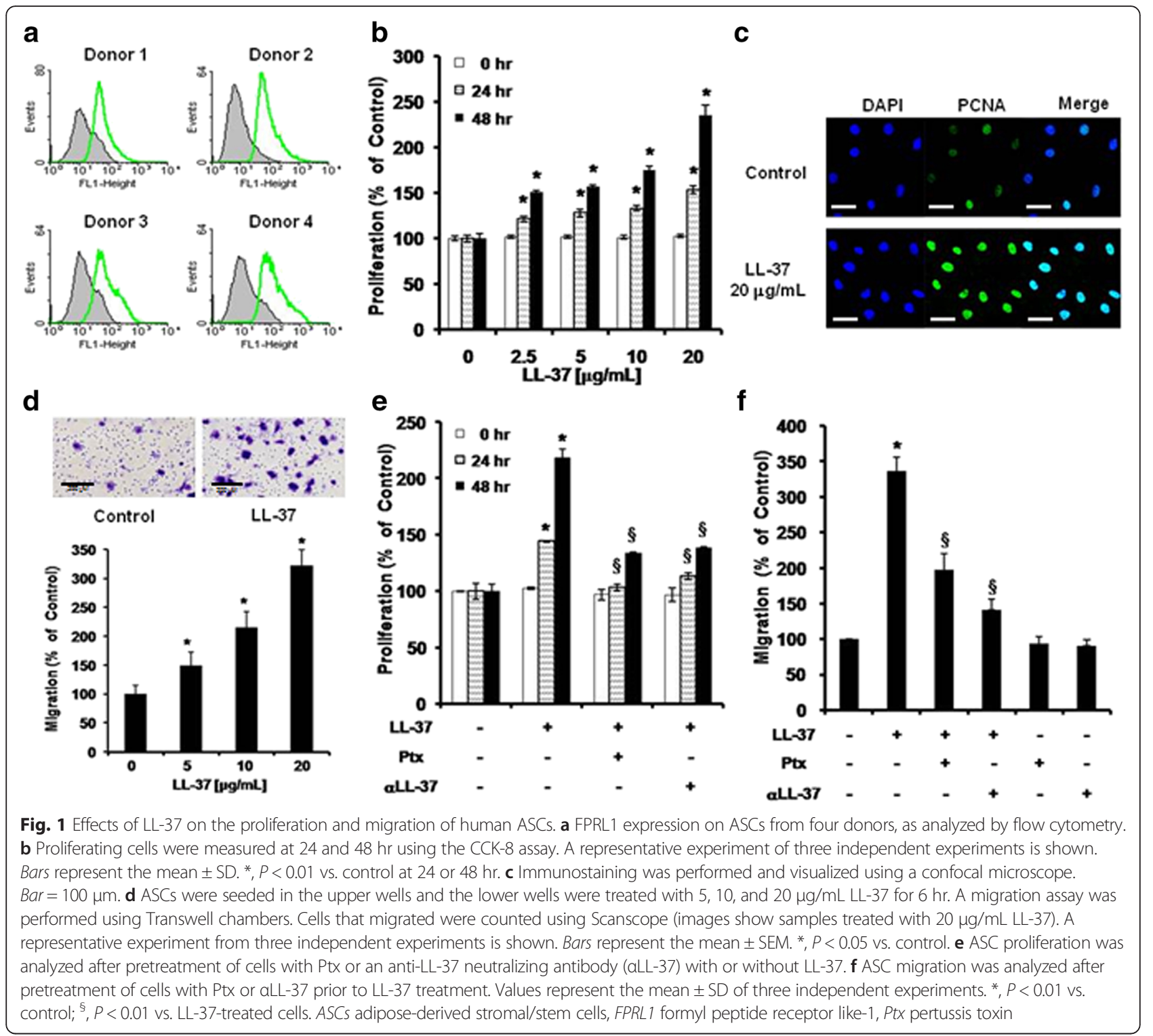

different amounts of time and then real-time PCR analysis was performed. LL-37 treatment significantly increased the EGR1 mRNA level, which peaked at $1 \mathrm{hr}$ and returned to the basal level at about $6 \mathrm{hr}$ (Fig. 2a). Because LL-37 treatment considerably increased the mRNA level of EGR1, we attempted to ascertain whether it also increased the protein level of EGR1 via western blot analysis. LL-37 treatment rapidly increased the protein level of EGR1 in ASCs in a time-dependent manner (Fig. 2b). In addition to western blot analysis, ELISA analysis of cell lysates demonstrated that EGR1 production was significantly increased by LL-37 treatment (Additional file 2: Figure S1b)

Next, to study the involvement of EGR1 in LL-37enhanced ASC proliferation and migration, ASCs were transfected with EGR1-targeting siRNA. We confirmed
EGR1 gene silencing at the protein level (Fig. 2c). LL-37enhanced proliferation and migration of ASCs were markedly attenuated by EGR1-targeting siRNA transfection (Fig. $2 \mathrm{~d}$ and e). These data suggest that EGR1 is important for LL-37-enhanced migration and proliferation of ASCs.

Involvement of the MAPK pathway in LL-37-induced ASC proliferation and migration

The MAPK pathway plays an important role in the migration and proliferation of MSCs and cancer cells $[10,28]$. To elucidate the signaling mechanism involved in LL-37-induced ASC proliferation and migration in detail, we examined the effect of LL-37 on the MAPK pathway in human ASCs. Treatment with LL-37 significantly increased the levels of phosphorylated 
Table 1 List of genes up-regulated by LL-37

\begin{tabular}{|c|c|c|c|}
\hline Gene name & $\begin{array}{l}\text { Accession } \\
\text { No }\end{array}$ & $\begin{array}{l}\text { Fold } \\
\text { increase }\end{array}$ & Classification \\
\hline Early growth response 1 (EGR1) & NM_001964 & 18.47 & Transcription, Immune response \\
\hline Early growth response 2 (EGR2) & NM_000399 & 6.13 & Transcription \\
\hline High mobility group AT-hook 2 (HMGA2) & NM_003483 & 4.72 & Transcription \\
\hline Response gene to complement 32 (RGC32) & NM_014059 & 4.33 & Cell cycle \\
\hline Solute carrier family 20 (phosphate transporter) (SLC20A1) & NM_005415 & 4.19 & Signal transduction \\
\hline Dual specificity phosphatase 1 (DUSP1) & NM_004417 & 3.94 & Cell cycle \\
\hline Dual specificity phosphatase 6 (DUSP6) & NM_001946 & 3.74 & Cell cycle, Signal transduction \\
\hline Connective tissue growth factor (CTGF) & NM_001901 & 3.71 & $\begin{array}{l}\text { Cell migration, Cell growth, Cell adhesion, Signal } \\
\text { transduction }\end{array}$ \\
\hline Kruppel-like factor 10 (KLF10) & NM_005655 & 3.31 & Transcription, Signal transduction, Cell proliferation \\
\hline Dachsous 1 (Drosophila) (DCHS1) & NM_003737 & 3.05 & Cell adhesion \\
\hline $\begin{array}{l}\text { Tumor necrosis factor receptor superfamily, member 12A } \\
\text { (TNFRSF12A) }\end{array}$ & NM_016639 & 2.98 & Apoptosis, Cell adhesion \\
\hline Pleckstrin homology-like domain, family A, member 2 (PHLDA2) & NM_003311 & 2.94 & Apoptosis \\
\hline v-fos FBJ murine osteosarcoma viral oncogene homolog (FOS) & NM_005252 & 2.80 & $\begin{array}{l}\text { Transcription, Inflammatory response, Immune } \\
\text { response }\end{array}$ \\
\hline Tribbles homolog 2 (Drosophila) (TRIB2) & NM_021643 & 2.56 & Cell adhesion \\
\hline Tubulin, beta 2C (TUBB2C) & NM_006088 & 2.52 & Transport, Apoptosis \\
\hline $\begin{array}{l}\text { Ras association (RalGDS/AF-6) and pleckstrin homology domains } 1 \\
\text { (RAPH1) }\end{array}$ & NM_213589 & 2.46 & Signal transduction \\
\hline Myeloid cell leukemia sequence 1 (BCL2-related) (MCL1) & NM_021960 & 2.34 & Apoptosis \\
\hline SH2B adaptor protein 3 (SH2B3) & NM_005475 & 2.26 & Cell cycle, Signal transduction \\
\hline Cysteine-rich, angiogenic inducer, 61 (CYR61) & NM_001554 & 2.19 & Cell adhesion, Cell proliferation \\
\hline Sphingosine kinase 1 (SPHK1) & NM_021972 & 2.10 & $\begin{array}{l}\text { Cell migration, Apoptosis, Cell cycle, Cell } \\
\text { proliferation }\end{array}$ \\
\hline $\begin{array}{l}\text { Ras association (RalGDS/AF-6) and pleckstrin homology domains } 1 \\
\text { (RAPH1) }\end{array}$ & NM_213589 & 2.01 & Signal transduction \\
\hline
\end{tabular}

The fold increase indicates the increase in expression in comparison to control cells, as determined by a human cDNA microarray

extracellular signal-regulated kinase (ERK) $1 / 2$, p38, and c-Jun N-terminal kinase (JNK), but did not change the total levels of these proteins (Fig. 3a). To further determine the involvement of kinase phosphorylation in the increased migration, proliferation, and EGR1 production of ASCs, cells were treated with LL-37 in the presence and absence of a specific inhibitor of ERK (PD98059), p38 (SB203580), or JNK (SP600125). All these MAPK inhibitors (PD98059, SB203580, and SP600125) significantly reduced LL-37-induced EGR1 production (Fig. 3b). In addition, each of the inhibitors attenuated the LL-37induced increase in ASC migration and proliferation (Fig. 3c and d). Taken together, these data suggest that: 1) LL-37 induces multiple signaling pathways, including those linked with ERK, p38, and JNK phosphorylation; and 2) these kinases are involved in the regulation of human ASC migration and proliferation. These results suggest that LL-37 stimulates ASC function through a MAPK-dependent mechanism.
LL-37 treatment enhances growth factor production in human ASCs, and treatment with the CM of ASCs pre-activated with LL-37 stimulates hair regeneration in vivo It was recently reported that EGR1 has a strong paracrine capability and can influence angiogenic factors in ASCs [8, 29]; therefore, we first examined whether LL37 regulates the expression of various regenerative growth factors and bioactive molecules in ASCs. LL-37 treatment considerably upregulated the mRNA expression of VEGF, TB4, MCP-1, and SDF-1 (Fig. 4a-d). We next confirmed the direct secretion of these growth factors by ASCs using ELISAs. The secretion levels of VEGF, TB4, MCP-1, and SDF-1 were significantly increased by LL-37 treatment (Fig. $4 \mathrm{e}-\mathrm{h}$ ).

To further evaluate the paracrine effects of LL-37 in vivo, the hair growth of C57BL/6 mice with hair loss was tested in vivo. We compared hair growth after topically applying CM of ASCs pre-activated with or without LL-37 $(20 \mu \mathrm{g} / \mathrm{mL})$. After 2 weeks, the hair growth 
a

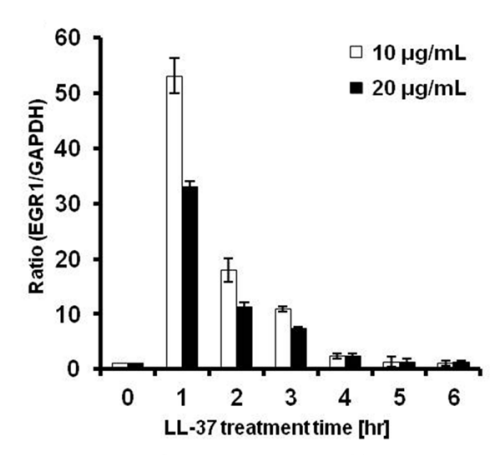

b
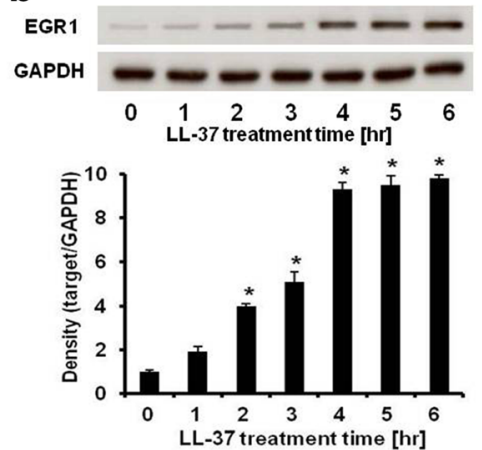
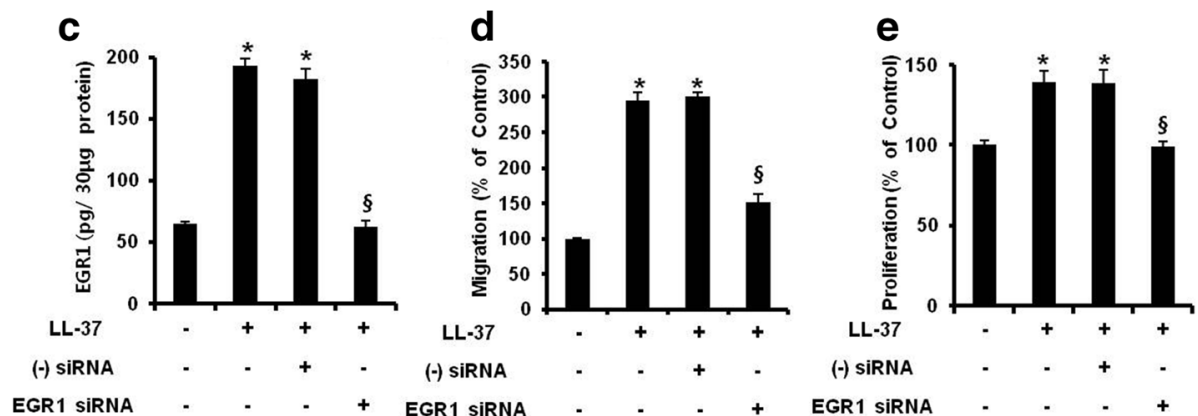

Fig. 2 EGR1 is critical for human LL-37-enhanced ASC migration and proliferation. a EGR1 mRNA expression was determined by real-time PCR. EGR1 mRNA was detected after treatment with 10 and $20 \mu \mathrm{g} / \mathrm{mL} \mathrm{LL}-37$ for 0-6 hr. b ASCs were incubated with $20 \mu \mathrm{g} / \mathrm{mL} \mathrm{LL}-37$ for 0-6 hr. Cell lysates were collected for western blot analysis. The EGR1 protein level was increased by LL-37 treatment in a time-dependent manner. c ASCS were treated with LL-37 and transfected with siRNA targeting EGR1 or a negative control sequence. After stabilization, cells were collected and lysed by the addition of $200 \mu \mathrm{L}$ of cell lysis buffer. EGR1-targeting siRNA transfection was quantified by performing an EGR1-specific ELISA of cell lysates. $\mathbf{d}$ Cells that migrated after EGR1-targeting siRNA transfection were imaged by microscopy and counted using Scanscope. Results are expressed as the mean \pm SD of three independent experiments. ${ }^{*}, P<0.01 \mathrm{vs}$. control; ${ }^{\S}, P<0.01 \mathrm{vs}$. LL-37-treated cells transfected with negative control siRNA. e The effects on human ASC proliferation were similar to those on migration. A representative experiment from three independent experiments is shown. Bars represent the mean $\pm \mathrm{SD}$. ${ }^{*}, P<0.01 \mathrm{vs.}$ control; ${ }^{\S}, P<0.01$ vs. LL-37-treated cells transfected with negative control siRNA. EGR1 early growth response 1, ASC adipose-derived stromal/stem cells, siRNA small interfering RNA, ELISA enzyme-linked immunosorbent assay

score was higher in the group treated with CM of ASCs than in the negative control group treated with medium alone. Moreover, the hair growth score was higher in the group treated with CM of ASCs pre-activated with LL37 than in the group treated with CM of non-activated ASCs (Fig. 4i and $j$ ), with dark pigmentation and hair regeneration observed on the initially pink hairless skin. Treatment with minoxidil (MNX), which is widely used to treat hair loss, was also effective for hair regeneration. Specifically, 95-100\% of hair was regenerated to full length in the group treated with CM of ASCs preactivated with LL-37 in comparison to the MNX-treated group, whereas the level of regeneration was lower in the group treated with CM of non-activated ASCs (30$35 \%$; Fig. 4i). Additionally, to elucidate the biological function of EGR1 in vitro and in vivo, EGR1 was lentivirally introduced into human ASCs. Cell lines stably expressing the control vector and EGR1 were designated pLenti6/V5-D-TOPO and pLenti6/V5-hEGR1, respectively.
As expected, the secretion levels of VEGF, TB4, MCP-1, and SDF-1 were significantly higher in the medium of cells overexpressing EGR1 than in the medium of wild type (WT) cells or those expressing the control vector (Additional file 2: Figure S2a-2d). To confirm the paracrine effects of stable EGR1-overexpressing cells in vivo, we performed an additional investigation of hair regeneration. Mice were treated with CM of WT ASCs, ASCs expressing the control vector, or EGR1-overexpressing ASCs. Compared with CM of WT ASCs and ASCs expressing the control vector, treatment with $\mathrm{CM}$ of EGR1-overexpressing ASCs considerably increased hair regeneration (Additional file 2: Figure S2e and 2f).

Taken together, LL-37 might mediate paracrine actions by stimulating the secretion of growth factors such as VEGF, TB4, SDF-1, and MCP-1 by ASCs. In addition, CM from ASCs pre-activated with LL-37 and EGR1overexpressing cells strongly promotes hair growth in vivo. 


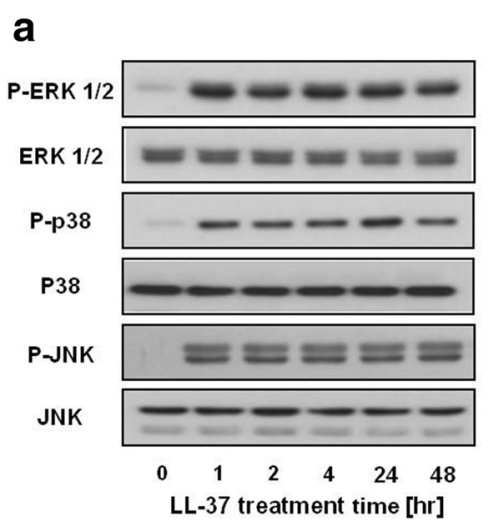

C

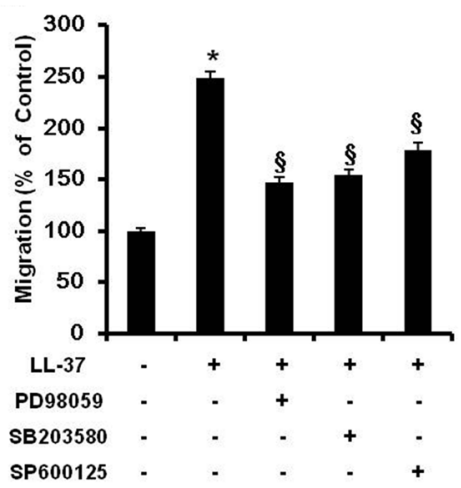

b

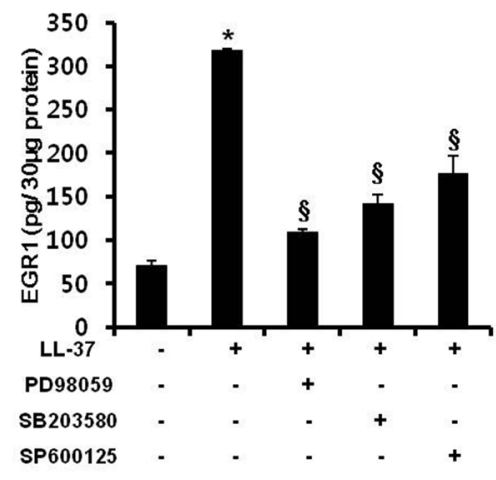

d

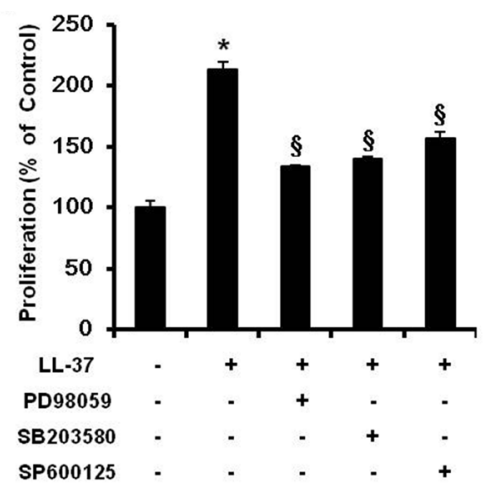

Fig. 3 Involvement of the MAPK pathway in LL-37-induced ASC proliferation and migration. a Human ASCs were treated with $20 \mu \mathrm{g} / \mathrm{mL} \mathrm{LL}-37$ for 1, 2, 4, 24, and $48 \mathrm{hr}$. After cell lysis, the levels of phosphorylated ERK1/2, p38, and JNK were determined by western blot analysis. The levels of total ERK1/2, p38, and JNK were used to confirm equal loading of the cell lysates. LL-37 treatment significantly increased the phosphorylation of ERK1/2, p38, and JNK. b ASCs were pretreated with or without a specific inhibitor of ERK1/2 (PD98059), p38 (SB203580), or JNK (SP600125) for 1 hr and then treated with $20 \mu \mathrm{g} / \mathrm{mL}$ LL-37. EGR1 protein levels in cell lysates were analyzed by an EGR1-specific ELISA. c LL-37-enhanced migration decreased in human ASCs treated with PD98059, SB203580, or SP600125. d LL-37-enhanced ASC proliferation was inhibited by treatment with specific inhibitors of ERK1/2, p38, or JNK (similar to ASC migration). A representative experiment from three independent experiments is shown. Bars represent the mean \pm SD. ${ }^{*}, P<0.01$ vs. control; ${ }^{\S}, P<0.01$ vs. LL-37-treated ASCS. MAPK mitogen-activated protein kinase, ASCs adipose-derived stromal/stem cells, EGR1 early growth response-1

\section{Discussion}

Many recent studies report clinical trials of ASCs (or CM from ASCs) for the treatment of vascular injury and for the promotion of bone, skin, and hair regeneration $[1,2,30]$. However, the regenerative effects of MSCs are limited because they vary according to the donor, the donor's age, the sampling site, and the culture techniques and conditions. Therefore, it is essential to develop an activator of MSCs. LL-37 is a naturally occurring antimicrobial peptide found in the wound bed, where it assists wound repair and regeneration [15-17]; it is important to elucidate the relationship between LL-37 and adjacent ASCs for tissue regeneration and repair. Therefore, we investigated the functions of ASCs activated by LL-37 exposure.

We demonstrated that human ASCs expressed FPRL1 and that LL-37 treatment significantly enhanced the proliferation and migration of these cells. Next, we searched for the factor(s) that underlies LL-37-enhanced migration and proliferation of ASCs. Initially, we thought that TB4 and MCP-1 were candidates for such factors because LL37 treatment considerably enhanced their production (Fig. 4f and h), which facilitated ASC migration (unpublished data). However, these are not the main factors, although this is only one possible mechanism underlying LL-37-enhanced migration of ASCs. This is because LL37 treatment rapidly promoted the migration of human ASCs within $6 \mathrm{hr}$ (Fig. 1d), whereas TB4 and MCP-1 production was noticeably changed much later, after about $24 \mathrm{hr}$ (Fig. $4 \mathrm{f}$ and h). Our microarray and real-time PCR data showed that EGR1 mRNA expression rapidly responded to LL-37 stimulation within $1 \mathrm{hr}$ (Fig. 2a and Table 1). Moreover, western blot and ELISA analyses revealed that LL-37 treatment also significantly increased EGR1 protein levels by about 5-10-fold at $6 \mathrm{hr}$ (Fig. 2b and Additional file 2: Figure S1b), which regulated ASC migration (Fig. 2d). Some previous studies reported that EGR1 mRNA was rapidly induced within 1-2 hr and that 

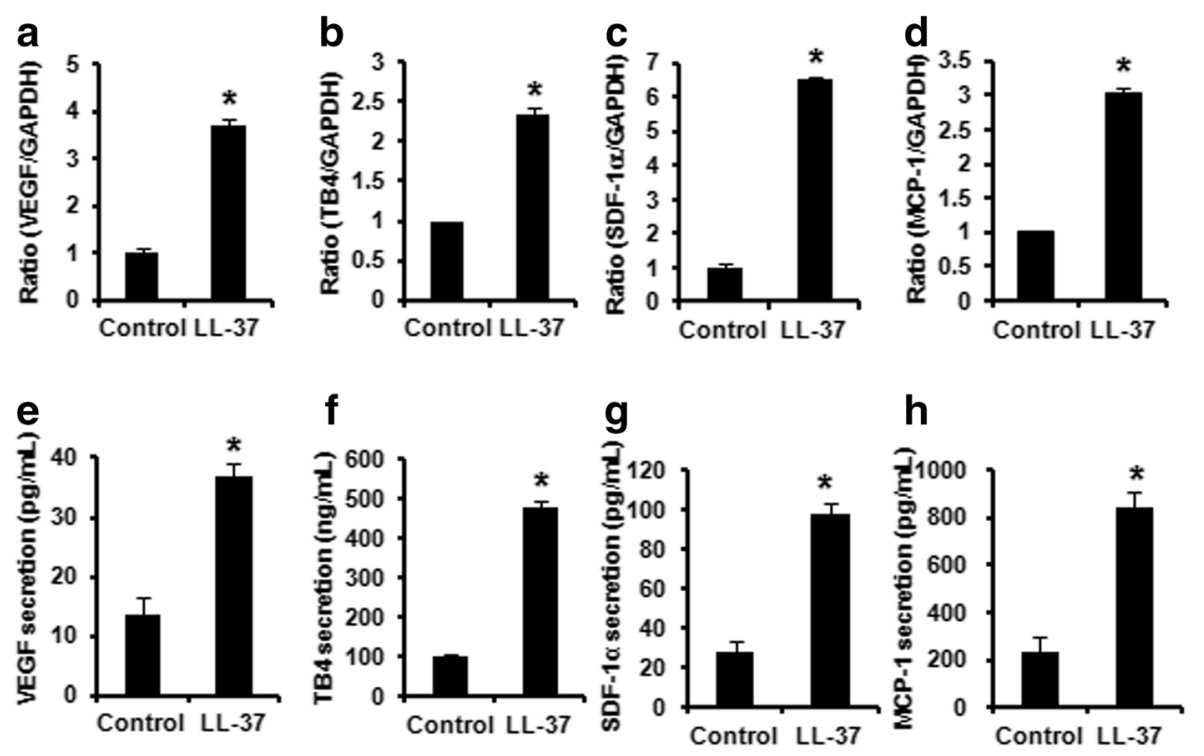

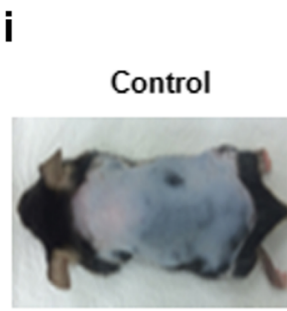

ASC-LL-37

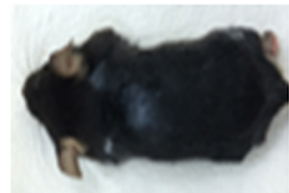

ASC-CM

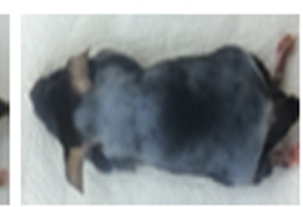

MNX

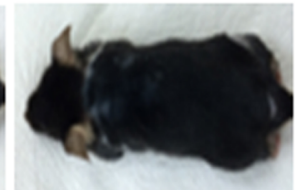

j

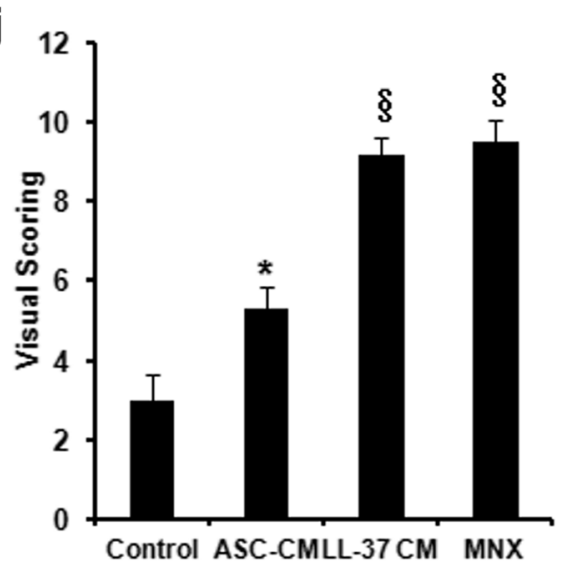

Fig. 4 Preconditioning with LL-37 stimulates hair regeneration in vivo and production of regenerative factors in vitro. a VEGF, (b) TB4, (c) SDF-1a, and (d) MCP-1 mRNA expression was determined by real-time PCR. e VEGF, (f) TB4, (g) SDF-1a, and (h) MCP-1 proteins were detected using the supernatants of confluent cell cultures. ASCs were treated with $20 \mathrm{\mu g} / \mathrm{mL} \mathrm{LL}-37$ for $48 \mathrm{hr}$, and then the protein levels of VEGF, TB4, SDF-1a, and MCP-1 were analyzed using specific ELISAs. A representative experiment from three independent experiments is shown. Bars represent the mean \pm SD. ${ }^{*}, P<0.05$ vs. control. i Hair was removed from the backs of C57BL/6 mice and the hair growth rate was monitored for 3 weeks. CM of human ASCs pretreated with or without LL-37 $(20 \mu \mathrm{g} / \mathrm{mL})$ was topically applied daily for up to 18 days to mice with hair loss. Gross views observed by photographs. $\mathbf{j}$ Hair growth was scored as described in the Materials and methods section. *, $P<0.05$ for control vs. group treated with ASC CM. ${ }^{\S}, P<0.05$ for group treated with ASC CM vs. group treated with CM of ASCs pre-activated with LL-37. VEGF vascular endothelial growth factor, TB4 thymosin beta-4, SDF-1a stromal cell-derived factor-1a, MCP-1 monocyte chemoattractant protein-1, ASC adipose-derived stromal/stem cell, ELISA enzyme-linked immunosorbent assay, CM conditioned medium

EGR1 protein was also relatively rapidly induced at the same time [29, 31]. Interestingly, our data showed that EGR1 protein induction was a little sluggish, although EGR1 mRNA expression was immediately increased within $1 \mathrm{hr}$. However, as in other reports, EGR1 protein was slowly induced at 5-6 hr [32-34]. It is possible that the optimal time of EGR1 induction differs according to the type of stimulus (e.g., cytokine, growth factor, and oxidative stress) or the cell type. A recent study by Min et al. suggested that EGR1 controls hematopoietic stem cell migration and expansion [35]. Similarly, our data showed that EGR1-targeting siRNA transfection inhibited LL-37enhanced proliferation and migration of ASCs (Fig. 2c-e). Thus, these results suggest that EGR1 is important for the regulation of LL-37-enhanced proliferation and migration of human ASCs.

In this study, LL-37 promoted the expansion and migration of human ASCs at all concentrations, but was most effective at $20 \mu \mathrm{g} / \mathrm{mL}$ (Fig. $1 \mathrm{~b}$ and d). Interestingly, EGR1 expression was highest in ASCs treated with a lower concentration $(10 \mu \mathrm{g} / \mathrm{mL})$ of LL-37 (Fig. 2a), suggesting that, in addition to EGR1, other factors and 
mechanisms mediate the effects of LL-37 on the proliferation and migration of human ASCs. For example, LL-37 treatment also considerably increased interleukin (IL)-8 expression and secretion in human ASCs (Additional file 2: Figure S3a and S3b). We previously demonstrated that IL-8 induced by TB4 plays a key role in ASC proliferation [23]. Moreover, IL-8 stimulated ASC migration, and IL-8 knockdown using IL-8-specific siRNA inhibited cell migration (unpublished data). IL-8 promotes angiogenesis and migration of bone marrow-derived MSCs [36], and may also affect multiple functions of human ASCs, including their proliferation and migration. Further investigations are necessary to clarify the other mechanisms underlying LL-37-induced proliferation and migration of human ASCs and the factors involved. Taken together, these data suggest that EGR1 is an important, but not the only, factor for LL-37-mediated ASC migration and proliferation.

It was recently reported that EGR1 has a strong paracrine capability and can influence angiogenic factors in ASCs [8, 29]; therefore, we investigated the enhanced secretion of TB4, VEGF, MCP-1, and SDF-1 in response to LL-37 stimulation (Fig. 4a-h). These growth factors and cytokines are associated with the regeneration and repair of various tissues, including skin, bone, and skeletal muscle [37-40]. Among them, TB4 directly stimulates hair growth and proliferation of hair follicle dermal papilla cells. Furthermore, TB4 accelerates hair growth via activation, migration, and differentiation of hair follicle stem cells [38, 41]. Recently, VEGF was shown to stimulate hair growth by facilitating the supply of nutrients to the hair follicle, increasing the follicular diameter $[42,43]$. Based on these reports, TB4 and VEGF are regarded as the main factors in the hair growth promotion effect of the CM of LL-37-activated ASCs. Interestingly, one of these regenerative factors, MCP-1, is a pro-inflammatory cytokine. However, it also promotes healing in diabetic wounds, dental pulp, and skeletal muscle by increasing cell migration, angiogenesis, or macrophage infiltration, leading to a higher regenerative potential $[39,44,45]$. Therefore, MCP-1 seems to be a key cytokine mediating regenerative effects as well as a pro-inflammatory cytokine, and its action might depend on the microenvironment in the human body. LL-37-enhanced regenerative factors also influence their microenvironment through paracrine actions [46]. In support of this, CM from ASCs pre-activated with LL-37 strongly promoted hair growth as efficiently as MNX (95-100 \% of hair), which is a

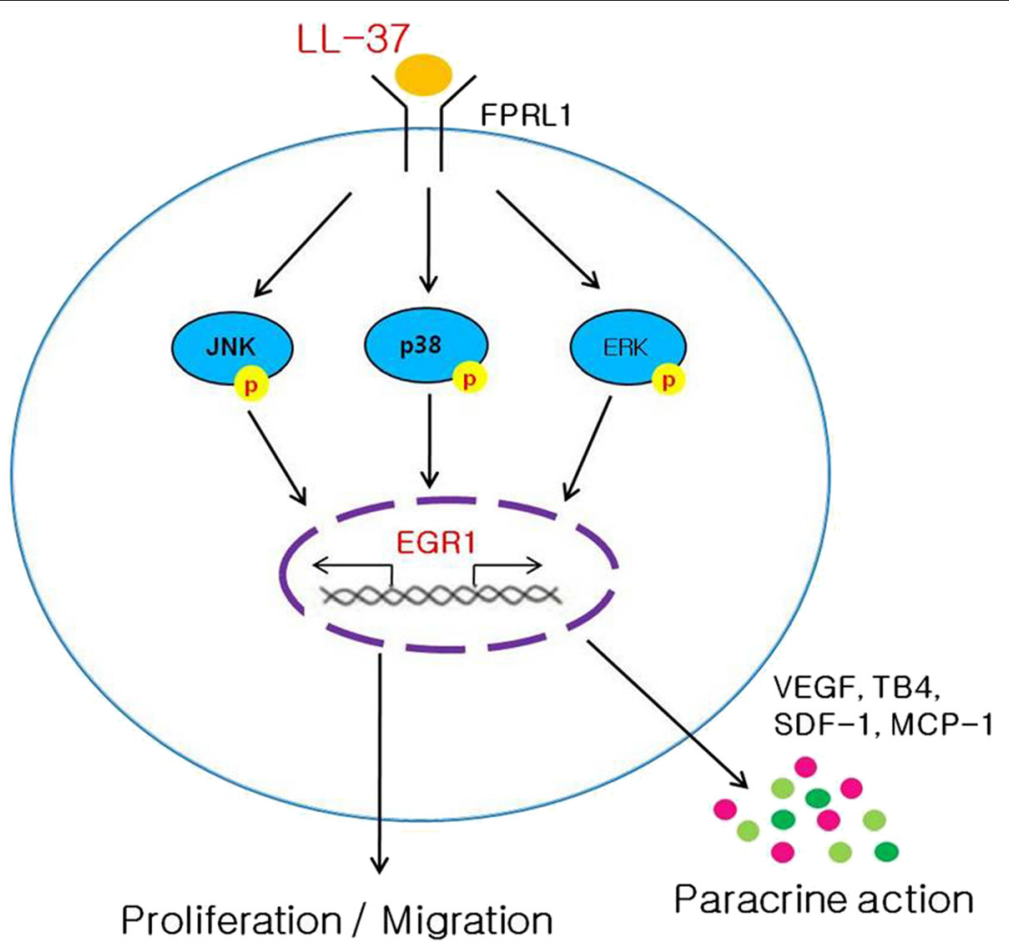

Fig. 5 Scheme representing the functions of ASCs after LL-37 treatment. LL-37 enhances ASC proliferation and migration via the EGR1 and MAPK pathways. Furthermore, LL-37 stimulates the secretion of growth factors such as VEGF, TB4, SDF-1, and MCP-1 in human ASCs. CM from ASCS preconditioned with LL-37 strongly promotes hair growth in vivo. LL-37 can be modulated to activate human ASCs and may provide a therapeutic approach for tissue regeneration by enforcing the functions of ASCs (e.g., expansion, migration, and paracrine actions). ASCs adipose-derived stromal/ stem cells, EGR1 early growth response 1, MAPK mitogen-activated protein kinase, VEGF vascular endothelial growth factor, TB4 thymosin beta-4, SDF-1a stromal cell-derived factor-1a, MCP-1 monocyte chemoattractant protein-1, CM conditioned medium 
commercially available treatment for hair loss (Fig. 4i and j). Besides these paracrine actions, we previously demonstrated that the SDF-1/CXCR4 axis is essential for human dermal fibroblast (HDF) migration, resulting in woundhealing effects [25]. This suggests that CXCR4-expressing HDFs can migrate along the concentration gradient of SDF-1 secreted by human ASCs in response to LL-37 at wound sites, leading to wound repair and regeneration. Liu et al. and Neuhaus et al. reported that SDF-1 also enhances EGR1 expression in endothelial cells and angiogenesis in ischemic regions $[47,48]$. Based on these reports, LL-37-induced SDF-1 might also enhance EGR1 expression in human ASCs. Therefore, it is reasonable to conclude that LL-37 can gradually accelerate other mechanisms via autocrine loops and paracrine actions on adjacent cells such as HDFs. Taken together, LL-37 may activate human ASCs via autocrine and paracrine actions.

\section{Conclusions}

In summary, these results demonstrate that LL-37 enhances ASC proliferation and migration via the EGR1 and MAPK pathways (Fig. 5). Furthermore, LL-37 stimulates the secretion of growth factors such as VEGF, TB4, SDF-1, and MCP-1. CM from ASCs preconditioned with LL-37 strongly promotes hair growth in vivo. Therefore, LL-37 can be modulated to activate human ASCs and may provide a therapeutic approach to tissue regeneration by enforcing the functions of ASCs (e.g., expansion, migration, and paracrine actions).

\section{Additional files}

Additional file 1: Table S1. List of primers used for real-time PCR. (PDF $106 \mathrm{~kb}$ )

Additional file 2: Figure S1. Effects of LL-37 on the viability and EGR1 production of human ASCs. Figure S2. EGR1-overexpressing ASCs secrete higher levels of the regenerative factors VEGF, TB4, SDF-1a, and MCP-1, and the CM of these cells stimulates hair regeneration in vivo. Figure S3. LL-37 increases the expression and production of IL-8 in human ASCs. (PDF $267 \mathrm{~kb}$ )

Additional file 3: Table S2. List of upregulated genes in ASCs exposed to LL-37 by microarray analysis. (XLS $45 \mathrm{~kb}$ )

\section{Abbreviations}

ASC: adipose-derived stromal/stem cell; CCK-8: cell counting kit-8; CM: conditioned medium; CTGF: connective tissue growth factor; EGR1: early growth response 1; EGR2: early growth response 2; ELISA: enzyme-linked immunosorbent assay; ERK: extracellular signal-regulated kinase; FACS: fluorescence-activated cell sorting; FPRL 1: formyl peptide receptor like-1; hCAP-18: human cationic antimicrobial protein 18; HDF: human dermal fibroblast; IL: interleukin; JNK: c-Jun N-terminal kinase; KLF10: Krupplelike factor 10; MAPK: mitogen-activated protein kinase; MCP-1: monocyte chemoattractant protein-1; MNX: minoxidil; MSC: mesenchymal stem cell; PCNA: proliferating cell nuclear antigen; Ptx: pertussis toxin; SDF-1: stromal cell-derived factor-1; siRNA: small interfering RNA; TB4: thymosin beta-4; VEGF: vascular endothelial growth factor; WT: wild type; aLL-37: anti-LL-37 antibody.

\section{Competing interests}

The authors declare that they have no competing interests.

\section{Authors' contributions}

YY contributed to conception and design of the study, data analysis and interpretation, collection and assembly of data, and drafting and revision of the manuscript. HC carried out the molecular work involving western blotting, siRNA transfection, and ELISAs as well as the animal work and contributed to drafting of the manuscript. MS performed the cell culture, characterization of ASCs, the migration assay, and the proliferation assay with statistical analysis, and participated in drafting of the manuscript. DC contributed to analysis and interpretation of the data, financial support, and helped to revise the manuscript. SB conceived and designed the study, provided financial support, and gave final approval of the manuscript. All authors read and approved the final manuscript.

\section{Acknowledgments}

We thank Jaehee Kim for support with the revision of the article and analysis of the data. This work was supported by a Samsung Biomedical Research Institute grant (SMO 1131631) and the Korea Drug Development Fund (KDDF), which is funded by the Ministry of Science, ICT and Future Planning, the Ministry of Trade, Industry \& Energy, and the Ministry of Health \& Welfare of the Republic of Korea (KDDF-201404-04).

\section{Author details}

'Department of Plastic Surgery, Samsung Medical Center, Sungkyunkwan University School of Medicine, Seoul, Korea. ${ }^{2}$ Department of Life Science, Sookmyung Women's University, Seoul, Korea. ${ }^{3}$ Bio-Med Translational Research Center, Samsung Medical Center, Seoul, Korea.

Received: 14 October 2015 Revised: 21 March 2016 Accepted: 4 April 2016 Published online: 19 April 2016

\section{References}

1. Bruder SP, Fink DJ, Caplan Al. Mesenchymal stem cells in bone development, bone repair, and skeletal regeneration therapy. J Cell Biochem. 1994;56(3):283-94.

2. Khosrotehrani K. Mesenchymal stem cell therapy in skin: why and what for? Exp Dermatol. 2013;22(5):307-10.

3. Zuk PA, Zhu M, Ashjian P, De Ugarte DA, Huang Jl, Mizuno H, et al. Human adipose tissue is a source of multipotent stem cells. Mol Biol Cell. 2002;13(12):4279-95.

4. Maijenburg MW, van der Schoot CE, Voermans C. Mesenchymal stromal cell migration: possibilities to improve cellular therapy. Stem Cells Dev. 2012;21(1):19-29.

5. Lee JW, Fang X, Krasnodembskaya A, Howard JP, Matthay MA. Concise review: mesenchymal stem cells for acute lung injury: role of paracrine soluble factors. Stem Cells. 2011;29(6):913-9.

6. Liang X, Ding Y, Zhang Y, Tse HF, Lian Q. Paracrine mechanisms of mesenchymal stem cell-based therapy: current status and perspectives. Cell Transplant. 2014;23(9):1045-59.

7. Tarcic G, Avraham R, Pines G, Amit I, Shay T, Lu Y, et al. EGR1 and the ERK-ERF axis drive mammary cell migration in response to EGF. FASEB J. 2012;26(4):1582-92.

8. Tamama K, Barbeau DJ. Early growth response genes signaling supports strong paracrine capability of mesenchymal stem cells. Stem Cells Int. 2012;2012:428403.

9. Caplan AI. Why are MSCs therapeutic? New data: new insight. J Pathol. 2009;217(2):318-24.

10. Ben-Chetrit N, Tarcic G, Yarden Y. ERK-ERF-EGR1, a novel switch underlying acquisition of a motile phenotype. Cell Adh Migr. 2013;7(1):33-7.

11. Murakami M, Ohtake T, Dorschner RA, Schittek B, Garbe C, Gallo RL. Cathelicidin anti-microbial peptide expression in sweat, an innate defense system for the skin. J Invest Dermatol. 2002;1 19(5):1090-5.

12. Frohm Nilsson M, Sandstedt B, Sorensen O, Weber G, Borregaard N, StahleBackdahl M. The human cationic antimicrobial protein (hCAP18), a peptide antibiotic, is widely expressed in human squamous epithelia and colocalizes with interleukin-6. Infect Immun. 1999;67(5):2561-6.

13. Larrick JW, Hirata M, Balint RF, Lee J, Zhong J, Wright SC. Human CAP18: a novel antimicrobial lipopolysaccharide-binding protein. Infect Immun. 1995;63(4):1291-7 
14. Niyonsaba F, Ushio $H$, Nagaoka I, Okumura K, Ogawa $H$. The human beta-defensins $(-1,-2,-3,-4)$ and cathelicidin LL-37 induce IL-18 secretion through p38 and ERK MAPK activation in primary human keratinocytes. J Immunol. 2005;175(3):1776-84.

15. Kai-Larsen $Y$, Agerberth B. The role of the multifunctional peptide LL-37 in host defense. Front Biosci. 2008;13:3760-7.

16. Carretero M, Escamez MJ, Garcia M, Duarte B, Holguin A, Retamosa L, et al. In vitro and in vivo wound healing-promoting activities of human cathelicidin LL-37. J Invest Dermatol. 2008;128(1):223-36.

17. Heilborn JD, Nilsson MF, Kratz G, Weber G, Sorensen O, Borregaard N, et al. The cathelicidin anti-microbial peptide LL-37 is involved in reepithelialization of human skin wounds and is lacking in chronic ulcer epithelium. J Invest Dermatol. 2003;120(3):379-89.

18. De Y, Chen Q, Schmidt AP, Anderson GM, Wang JM, Wooters J, et al. LL-37, the neutrophil granule- and epithelial cell-derived cathelicidin, utilizes formyl peptide receptor-like 1 (FPRL1) as a receptor to chemoattract human peripheral blood neutrophils, monocytes, and T cells. J Exp Med. 2000; 192(7):1069-74.

19. Krasnodembskaya A, Song Y, Fang X, Gupta N, Serikov V, Lee JW, et al. Antibacterial effect of human mesenchymal stem cells is mediated in part from secretion of the antimicrobial peptide LL-37. Stem Cells. 2010;28(12):2229-38.

20. Cashman TJ, Gouon-Evans V, Costa KD. Mesenchymal stem cells for cardiac therapy: practical challenges and potential mechanisms. Stem Cell Rev. 2013;9(3):254-65.

21. Pound LD, Patrick C, Eberhard CE, Mottawea W, Wang GS, Abujamel T, et al. Cathelicidin antimicrobial peptide: a novel regulator of islet function, islet regeneration, and selected gut bacteria. Diabetes. 2015;64(12):4135-47.

22. Kittaka M, Shiba H, Kajiya M, Fujita T, Iwata T, Rathvisal K, et al. The antimicrobial peptide LL37 promotes bone regeneration in a rat calvarial bone defect. Peptides. 2013;46:136-42.

23. Jeon BJ, Yang Y, Kyung Shim S, Yang HM, Cho D, Ik BS. Thymosin beta- 4 promotes mesenchymal stem cell proliferation via an interleukin-8-dependent mechanism. Exp Cell Res. 2013;319(17):2526-34.

24. Dominici M, Le Blanc K, Mueller I, Slaper-Cortenbach I, Marini F, Krause D, et al. Minimal criteria for defining multipotent mesenchymal stromal cells. The International Society for Cellular Therapy position statement. Cytotherapy. 2006:8(4):315-7.

25. Yang Y, Shim SK, Kim HA, Seon M, Yang E, Cho D, et al. CXC chemokine receptor 4 is essential for Lipo-PGE1-enhanced migration of human dermal fibroblasts. Exp Dermatol. 2012;21(1):75-7.

26. Vegesna V, O'Kelly J, Uskokovic M, Said J, Lemp N, Saitoh T, et al. Vitamin D3 analogs stimulate hair growth in nude mice. Endocrinology. 2002;143(11):4389-96.

27. Jung MK, Ha S, Huh SY, Park SB, Kim S, Yang Y, et al. Hair-growth stimulation by conditioned medium from vitamin D3-activated preadipocytes in C57BL/6 mice. Life Sci. 2015;128:39-46.

28. Yang Y, Cheon S, Jung MK, Song SB, Kim D, Kim HJ, et al. Interleukin-18 enhances breast cancer cell migration via down-regulation of claudin-12 and induction of the p38 MAPK pathway. Biochem Biophys Res Commun. 2015;459(3):379-86.

29. Kerpedjieva SS, Kim DS, Barbeau DJ, Tamama K. EGFR ligands drive multipotential stromal cells to produce multiple growth factors and cytokines via early growth response-1. Stem Cells Dev. 2012;21(13):2541-51.

30. Park BS, Kim WS, Choi JS, Kim HK, Won JH, Ohkubo F, et al. Hair growth stimulated by conditioned medium of adipose-derived stem cells is enhanced by hypoxia: evidence of increased growth factor secretion. Biomed Res. 2010;31(1):27-34.

31. Cheng JC, Chang HM, Leung PC. Egr-1 mediates epidermal growth factor-induced downregulation of E-cadherin expression via Slug in human ovarian cancer cells. Oncogene. 2013;32(8):1041-9.

32. Kwapiszewska G, Chwalek K, Marsh LM, Wygrecka M, Wilhelm J, Best J, et al. BDNF/TrkB signaling augments smooth muscle cell proliferation in pulmonary hypertension. Am J Pathol. 2012;181(6):2018-29.

33. Zhang $X$, Liu Y. Suppression of HGF receptor gene expression by oxidative stress is mediated through the interplay between Sp1 and Egr-1. Am J Physiol Renal Physiol. 2003;284(6):F1216-25.

34. Spohn D, Rossler OG, Philipp SE, Raubuch M, Kitajima S, Griesemer D, et al. Thapsigargin induces expression of activating transcription factor 3 in human keratinocytes involving $\mathrm{Ca} 2+$ ions and c-Jun N-terminal protein kinase. Mol Pharmacol. 2010;78(5):865-76.
35. Min IM, Pietramaggiori G, Kim FS, Passegue E, Stevenson KE, Wagers AJ. The transcription factor EGR1 controls both the proliferation and localization of hematopoietic stem cells. Cell Stem Cell. 2008;2(4):380-91.

36. Wang L, Li Y, Chen X, Chen J, Gautam SC, Xu Y, et al. MCP-1, MIP-1, IL-8 and ischemic cerebral tissue enhance human bone marrow stromal cell migration in interface culture. Hematology. 2002;7(2):113-7.

37. Liu YS, Ou ME, Liu H, Gu M, Lv LW, Fan C, et al. The effect of simvastatin on chemotactic capability of SDF-1alpha and the promotion of bone regeneration. Biomaterials. 2014;35(15):4489-98.

38. Philp D, Kleinman HK. Animal studies with thymosin beta, a multifunctional tissue repair and regeneration peptide. Ann N Y Acad Sci. 2010;1194:81-6.

39. Wood S, Jayaraman V, Huelsmann EJ, Bonish B, Burgad D, Sivaramakrishnan $\mathrm{G}$, et al. Pro-inflammatory chemokine CCL2 (MCP-1) promotes healing in diabetic wounds by restoring the macrophage response. PLoS One. 2014:9(3):e91574.

40. Niyaz M, Gurpinar OA, Oktar GL, Gunaydin S, Onur MA, Ozsin KK, et al. Effects of VEGF and MSCs on vascular regeneration in a trauma model in rats. Wound Repair Regen. 2015;23(2):262-7.

41. Philp D, St-Surin S, Cha HJ, Moon HS, Kleinman HK, Elkin M. Thymosin beta 4 induces hair growth via stem cell migration and differentiation. Ann N Y Acad Sci. 2007;1112:95-103.

42. Bassino E, Gasparri F, Giannini V, Munaron L. Paracrine crosstalk between human hair follicle dermal papilla cells and microvascular endothelial cells. Exp Dermatol. 2015;24(5):388-90.

43. Gnann LA, Castro RF, Azzalis LA, Feder D, Perazzo FF, Pereira EC, et al. Hematological and hepatic effects of vascular epidermal growth factor (VEGF) used to stimulate hair growth in an animal model. BMC Dermatol. 2013;13:15.

44. Hayashi Y, Murakami M, Kawamura R, Ishizaka R, Fukuta O, Nakashima M. CXCL14 and MCP1 are potent trophic factors associated with cell migration and angiogenesis leading to higher regenerative potential of dental pulp side population cells. Stem Cell Res Ther. 2015;6:111.

45. Zhang J, Xiao Z, Qu C, Cui W, Wang X, Du J. CD8 T cells are involved in skeletal muscle regeneration through facilitating MCP-1 secretion and Gr1(high) macrophage infiltration. J Immunol. 2014;193(10):5149-60.

46. Sassoli C, Pini A, Chellini F, Mazzanti B, Nistri S, Nosi D, et al. Bone marrow mesenchymal stromal cells stimulate skeletal myoblast proliferation through the paracrine release of VEGF. PLoS One. 2012;7(7):e37512.

47. Neuhaus T, Stier S, Totzke G, Gruenewald E, Fronhoffs S, Sachinidis A, et al. Stromal cell-derived factor 1alpha (SDF-1alpha) induces gene-expression of early growth response-1 (Egr-1) and VEGF in human arterial endothelial cells and enhances VEGF induced cell proliferation. Cell Prolif. 2003;36(2):75-86.

48. Liu H, Liu S, Li Y, Wang X, Xue W, Ge G, et al. The Role of SDF-1-CXCR4/ CXCR7 Axis in the Therapeutic Effects of Hypoxia-Preconditioned Mesenchymal Stem Cells for Renal Ischemia/Reperfusion Injury. PLoS ONE. 2012:7(4):e34608.

\section{Submit your next manuscript to BioMed Central and we will help you at every step:}

- We accept pre-submission inquiries

- Our selector tool helps you to find the most relevant journal

- We provide round the clock customer support

- Convenient online submission

- Thorough peer review

- Inclusion in PubMed and all major indexing services

- Maximum visibility for your research

Submit your manuscript at www.biomedcentral.com/submit 